\title{
Pensamento algébrico: contributo da visualização na construção da
}

generalização

\author{
Algebraic Thinking: contribution of visualization in the construction of \\ generalization
}

ISABEL VALE $^{1}$

ANA BARBOSA ${ }^{2}$

\begin{abstract}
Resumo
Para que os alunos sejam competentes em álgebra, devem compreender os conceitos $e$ relações muito para além da manipulação simbólica, o que implica que o seu estudo se inicie nos primeiros anos com o desenvolvimento do pensamento algébrico. Naturalmente, a formação dos futuros professores deve acompanhar esta tendência. Assim, apresenta-se parte de um estudo de natureza qualitativa, no qual se procura caracterizar o pensamento algébrico de futuros professores do ensino básico (3-12 anos) na resolução de tarefas envolvendo padrões figurativos. Os resultados mostraram que os participantes usaram estratégias visuais e analíticas, tendo prevalecido as primeiras, e que as maiores dificuldades surgiram nas questões de generalização distante, frequentemente influenciadas pelo tipo de estratégias usadas.
\end{abstract}

Palavras-chave: Generalização, Visualização, Pensamento Algébrico.

\begin{abstract}
For students to be competent in algebra, they must understand concepts and relationships far beyond mere symbolic manipulation, which implies that its study begins in the early years with the development of algebraic thinking, where the search for patterns and generalization in figurative contexts play a crucial role. Thus, we present part of a qualitative study, which seeks to analyze the algebraic thinking of future primary school teachers (3-12 years) in solving tasks involving figurative patterns. The results showed that the participants used visual and analytical strategies, having prevailed the former, and that the biggest difficulties arose in distant generalization tasks, often influenced by the type of strategies used.
\end{abstract}

Keywords: Generalization, Visualization, Algebraic Thinking.

\footnotetext{
1 Doutora em Didática pela Universidade de Aveiro (UA). Professora Coordenadora do Instituto Politécnico de Viana do Castelo, Portugal. E-mail: isabel.vale@ese.ipvc.pt

2 Doutora em Estudos da Criança-Matemática Elementar pela Universidade do Minho. Professora Adjunta do Instituto Politécnico de Viana do Castelo, Portugal. E-mail: anabarbosa@ese.ipvc.pt
} 


\section{Introdução}

A álgebra praticamente não tem expressão nos currículos de matemática dos primeiros anos do ensino básico, por se considerar a aritmética um tema mais fácil e, por isso, a álgebra deveria surgir mais tarde no currículo. No entanto, nas últimas décadas realizaram-se vários estudos a nível internacional que defendiam a introdução da álgebra a partir dos primeiros anos (KAPUT, 1998), com a algebrização do currículo da educação básica, tendo também surgido documentos curriculares que propõem as mesma ideias (NCTM, 2000). Estas recomendações vão para além da visão generalizada de que álgebra seria uma mera manipulação de símbolos algébricos que permitia resolver umas quantas tarefas rotineiras. Começou a defender-se que a álgebra é muito mais do que simbolismos, dando maior relevo ao pensamento algébrico como uma forma de pensamento matemático poderosa, acessível a alunos mais jovens. Este tipo de trabalho passa pela proposta de um conjunto de experiências matemáticas significativas, como identificar padrões, formular conjeturas e generalizações, pensar no particular e no geral, pensar de modo relacional e funcional entre números e operações, dar sentido ao simbolismo e à formalização de estruturas matemáticas (KIERAN, 2011; MOLINA, 2011; RADFORD, 2014).

Neste contexto, os professores têm um papel fundamental na abordagem da álgebra nos primeiros anos, mas é pertinente realçar que, por norma, as suas experiências pessoais com a álgebra escolar tradicional influenciam significativamente a sua perspectiva, limitando a abordagem que usam com os seus alunos. Muitos estudos com professores e futuros professores têm mostrado que o seu conhecimento sobre conceitos algébricos é muitas vezes fragmentado e consiste num sistema de símbolos e procedimentos sem conexão entre si (BALL, 1990; MA, 1999; PIMENTEL, 2011). Um ensino eficaz da álgebra requer uma preparação cuidadosa por parte dos professores ao longo da sua formação, isto implica que tenham as suas próprias experiências com uma álgebra mais rica, em que as conexões são contempladas, e compreender de que forma podem construir oportunidades dessa natureza com os seus alunos (BLANTON; KAPUT, 2005; NCTM, 2014). Neste sentido, é desejável que os professores desenvolvam uma profunda compreensão da matemática que vão ensinar, revisitando, com um outro olhar, conceitos já aprendidos, tendo oportunidade de experienciar as mesmas tarefas que irão utilizar com os seus alunos, de modo a que possam refletir sobre como orientar o questionamento, para promover um pensamento crítico e flexível. 
Reforçando a importância do pensamento algébrico, desenvolvemos um projeto que evidenciou em particular a pertinência da utilização de tarefas que envolvem a descoberta e o estudo de padrões em contextos figurativos/visuais, como componente essencial da construção da generalização (BARBOSA, 2013; PIMENTEL, 2011; VALE, 2009). Os diferentes trabalhos que temos desenvolvido (BARBOSA; VALE, 2015; VALE; PIMENTEL; BARBOSA, 2015; VALE; PIMENTEL; BARBOSA, 2018) permitem identificar que tradicionalmente os alunos são mais analíticos, mas há igualmente alunos visuais, mas também constatamos que pensar visualmente, ou procurar ver é passível de ser ensinado. Evidenciam, também, que uma abordagem desta natureza permite motivar alunos e professores para a matemática, desenvolver capacidades de ordem superior e estimular a sua criatividade, valorizando relações entre diferentes temas matemáticos, em contextos diversificados.

Tendo por base as ideias previamente discutidas, estamos interessadas no desenvolvimento do pensamento algébrico através do estudo de padrões figurativos (os termos são representados sob a forma de figuras), em particular os de crescimento (cada termo muda de forma previsível em relação ao anterior). Este tipo de tarefas facilita a formulação de conjeturas e a expressão da generalização, emergentes do raciocínio indutivo, que é acessível a alunos do ensino básico. Para além disso, fomentam o estabelecimento de conexões entre vários modos de representação. Essa articulação permite uma melhor compreensão da estrutura matemática subjacente, conduzindo, de modo mais eficaz, à conjetura, à generalização e à argumentação.

\section{Dos padrões ao pensamento algébrico}

A comunidade de educadores matemáticos tem promovido intensas discussões sobre o tipo de abordagem instrucional que poderá ter maior potencial na promoção da aprendizagem de conceitos algébricos. Há uma concordância no sentido de se defender

a integração da Álgebra desde os primeiros anos com ênfase em experiências que permitam aos alunos "aprender álgebra como um conjunto de conceitos e competências ligadas à representação de relações quantitativas e como uma forma de pensamento para formalizar padrões, funções e generalizações" (NCTM, 2000, p. 223). Na verdade, o estudo e compreensão da matemática requer que os alunos tenham experiências que vão para além do foco na aritmética e da fluência de cálculo, de modo a poderem desenvolver uma compreensão conceptual (BLANTON; KAPUT, 2011; NCTM, 2014). 
Isto implica que sejam capazes de reconhecer e articular estruturas e relações e de usar essas percepções do raciocínio matemático como objetos para raciocinar matematicamente. Na matemática elementar, este tipo de experiências relaciona-se com a emergência do pensamento algébrico, proporcionando a compreensão da generalidade, associada à matemática como um todo e não apenas à exploração de situações de aprendizagem computacionais (BARBOSA, 2013).

Apesar destas recomendações, a transição da aritmética para o estudo de uma matemática mais avançada não se afigura fácil para os alunos. E, por isso, a investigação acerca do ensino da álgebra nos primeiros anos (BLANTON; KAPUT, 2011; KIERAN, 2011; RADFORD, 2014) mostra que o trabalho com propostas que fomentem conexões ricas entre as ideias da aritmética e da álgebra podem facilitar a transição referida.

$\mathrm{Na}$ literatura é possível observar a atribuição de diferentes conotações ao termo pensamento algébrico, sendo, por isso, importante delimitá-lo. Swafford e Langrall (2000) consideram ser a capacidade de pensar em quantidades desconhecidas como sendo conhecidas. Kieran (2011) associa-o à utilização de uma diversidade de representações para analisar situações quantitativas de um modo relacional. Envolve o desenvolvimento de modos de pensar através de atividades para as quais o simbolismo da álgebra pode ser usado como ferramenta, mas que não são exclusivas da álgebra, podendo ser abordadas sem qualquer uso de simbolismo algébrico. Radford (2014) define pensamento algébrico como uma forma de reflexão e ação matemática, destacando a necessidade de se reconhecer formas elementares desse pensamento que não sejam exclusivamente baseadas no simbolismo alfanumérico. Kaput (2008) associa ao pensamento algébrico dois aspetos essenciais: (1) formular e expressar generalizações de modos gradualmente mais formais e convencionais; e (2) raciocinar com representações simbólicas, incluindo a sua manipulação. O mesmo autor destaca que estes aspetos integram três abordagens longitudinais da álgebra escolar: (1) a Álgebra como o estudo das estruturas e sistemas generalizados dos cálculos e das relações numéricas (aritmética generalizada); (2) a Álgebra como o estudo de funções, relações e variações (relações funcionais); e (3) a sua aplicação em situações de modelação, para exprimir e formalizar generalizações. Em termos gerais, destacamos a definição de Blanton e Kaput (2005) que consideram pensamento algébrico como "um processo no qual os alunos generalizam ideias matemáticas a partir de um conjunto de casos particulares, estabelecendo essas generalizações através do discurso da 
argumentação, expressando-as gradualmente de modos formais e apropriados à idade" (p. 413). Alguns investigadores (RADFORD, 2014; RIVERA 2011) sublinham ainda como ideias centrais a consciência da existência de padrões e de estrutura. Independentemente da definição usada, os princípios básicos do pensamento algébrico contemplam a construção da generalidade ao longo do currículo.

Percebe-se das ideias anteriores que o pensamento algébrico pode assumir diversas formas, entre elas encontra-se o raciocínio funcional, que tem sido identificado como um dos principais eixos deste tipo de pensamento (KAPUT, 2008; WARREN, 2008) e um dos conteúdos centrais na investigação em álgebra nos primeiros anos. Pode ser definido como o processo de pensamento utilizado na construção e generalização de padrões e relações, usando diversas ferramentas linguísticas e representacionais, explorando as relações generalizadas ou funções, que constituem objetos matemáticos por si próprias (BLANTON, 2008). Pensando na abordagem da álgebra por alunos mais jovens, pode dizer-se que a compreensão intuitiva do conceito de função pelas crianças começa a desenvolver-se antes da introdução formal das funções e o professor pode ajudar os alunos a desenvolver o raciocínio funcional, por exemplo, através de tarefas que impliquem a descoberta e generalização de padrões, facilitando a construção do conceito de função. A capacidade de reconhecer padrões e reorganizar dados para representar situações nas quais o input se relaciona com o output, através de regras funcionais bem definidas, é crítica para o desenvolvimento do pensamento algébrico (BLANTON; KAPUT, 2011; NCTM, 2014). A exploração de padrões constitui assim um meio privilegiado para introduzir a álgebra devido à possibilidade da representação dinâmica das variáveis envolvidas.

Para muitos autores a generalização de padrões está no coração da matemática (MASON, 1996). A procura de padrões tem vindo a ser associada à generalização, considerando-se que poderá conduzir naturalmente à expressão da generalidade (MASON, 1996). Este tipo de atividade matemática poderá ser um veículo poderoso para a compreensão de relações entre quantidades subjacentes às funções matemáticas, contribuindo assim para o estabelecimento de relações de tipo funcional (BARBOSA, 2013; BLANTON; KAPUT, 2011; WARREN, 2008). As tarefas de generalização de padrões são frequentemente usadas em sala de aula para envolver os alunos na identificação de um padrão, na sua extensão para encontrar o valor de um termo próximo ou distante e posterior articulação com a relação funcional subjacente ao 
padrão usando símbolos. Pode assim dizer-se que são processos fundamentais na promoção do pensamento algébrico.

\section{A visualização no pensamento algébrico}

A visualização tem um papel crucial na sociedade atual. A necessidade de pensar e raciocinar visualmente é muito forte e pode ser uma ferramenta cognitiva muito importante no desenvolvimento de conceitos e processos matemáticos, incluindo a resolução de problemas (RIVERA, 2011). Para Krutetskii (1976) há dois modos de pensamento matemático a que um indivíduo recorre, o lógico-verbal e o visualpictórico. Segundo este autor, é o equilíbrio entre estes dois modos de pensar que determina a forma como se relacionam as ideias matemáticas. Também Gardner (1993) afirma que alguns indivíduos reconhecem regularidades espacial ou visualmente, enquanto outros as percebem lógica ou analiticamente.

Assim, vários autores (BORROMEO-FERRI, 2015; KRUTETSKII, 1976; PRESMEG, 2014) consideram três tipos de alunos, dependendo de sua preferência de pensamento na resolução de tarefas matemáticos: (a) Analíticos (verbais) - aqueles alunos que recorrem ao uso de métodos de resolução não visuais, preferindo usar a processos de pensamento lógico-verbais, que envolvem representações algébricas, numéricas e verbais, mesmo com tarefas que seriam mais facilmente resolvidas através de uma abordagem visual; (b) Visuais (geométricos) - aqueles alunos que preferem usar métodos de resolução visuais, usando processos de pensamento visuais-pictóricos, que envolvem representações gráficas (e.g. figuras, diagramas, quadros), mesmo quando as tarefas seriam mais facilmente resolvidas por meios analíticos; e (c) Mistos (integrados) - aqueles alunos que não têm preferência por nenhum método de pensamento, quer seja lógico-verbal ou visual-pictórico. Têm um estilo de pensamento integrado porque combinam raciocínio analítico e visual.

O modo como o aluno processa a informação durante a sua aprendizagem tem grandes implicações para o professor na prática da sala de aula. De facto, é comum, que, em tarefas matemáticas, indivíduos diferentes processem a mesma informação de maneiras diferentes e, neste sentido, muitos alunos mostram preferência por métodos analíticos, enquanto outros tendem a raciocinar visualmente. E, por isso, o professor deve diversificar as suas abordagens para atender a esta diversidade. No âmbito da Álgebra, as tarefas de exploração de padrões podem ser propostas numa diversidade de contextos 
(e.g. numérico, geométrico, figurativo), no entanto a literatura indica que o recurso a um suporte visual pode conduzir à aplicação de estratégias de natureza diferente na tentativa de chegar à generalização, sejam elas de natureza visual ou analítica (ARCAVI, 2003; BARBOSA; VALE, 2015; RADFORD, 2014; RIVERA; 2011; VALE; PIMENTEL; BARBOSA, 2018). Em concordância com estas ideias, Gianquito (2007) defende que a identificação da estrutura subjacente a um padrão dá-se a partir da associação de teorias, conduzindo à utilização de abordagens analíticas, ou através da utilização de um modelo visual perceptível. Neste último caso existe a expectativa de ocorrência de um processo de projeção percepção-conceito, relacionado com a componente visual, que atua como suporte na construção ou contextualização dos elementos abstratos envolvidos (RIVERA, 2011). Reforçando esta ideia, e de acordo com o sentido atribuído por Arcavi (2003) à visualização em matemática, o recurso a modelos visuais como um tipo de estratégia permite que os alunos visualizem o invisível de um mundo abstrato dominado por relacionamentos e estruturas conceptuais que nem sempre são diretamente evidentes. Assim, ver é uma estratégia importante para ajudar na compreensão de conceitos matemáticos e pode ser desenvolvida através de experiências que requerem esse tipo de pensamento, mesmo para alunos que são mais visuais (VALE; PIMENTEL; BARBOSA, 2018).

Independentemente da abordagem adotada, a possibilidade de recorrer a uma grande diversidade de estratégias na tentativa de generalizar um padrão, permite reforçar a fluência representacional, enquanto capacidade de trabalhar com diferentes representações e de transitar eficazmente de umas para as outras (BIEDA; NATHAN, 2009). Nesta sequência de ideias é perceptível a relevância indiscutível dos contextos figurativos ou visuais. Uma imagem visual permite traduzir grande parte das informações relacionadas com determinada situação, facilitando a compreensão e a formulação mais rápida de argumentos sobre um dado conceito do que através de uma sequência de palavras (VALE; PIMENTEL; BARBOSA, 2015).

\section{Padrões e generalização em contextos visuais}

As estratégias na generalização de um padrão num contexto figurativo variam entre visuais e analíticas. Nas estratégias visuais, as figuras desempenham um papel fundamental na descoberta do invariante, enquanto que nas analíticas o trabalho desenvolvido tem foco nos números que emergem das figuras (BARBOSA; VALE, 
2015). Interessa agora discutir aspetos específicos relacionados com a aplicação de algumas das estratégias habitualmente utilizadas pelos alunos.

A literatura evidencia que os alunos optam por uma estratégia recursiva quando as tarefas de generalização "fornecem uma conexão clara com a mudança incremental" (LANNIN; BARKER; TOWNSEND, 2006, p.12), sejam propostas em contextos visuais ou não visuais. Essa conexão é frequentemente estabelecida quando os inputs são relativamente próximos (LANNIN et al., 2006). Isto explica-se porque a abordagem recursiva permite que os termos seguintes sejam determinados sem grande dificuldade. Esta abordagem é muito utilizada, especialmente pelos alunos mais novos ou por alunos que exibem mais dificuldades (BARBOSA; VALE, 2015; BLANTON; KAPUT, 2011; PIMENTEL, 2011; RIVERA; BECKER, 2008). No entanto, apesar de esta estratégia ser útil para determinar rapidamente os termos seguintes, uma das suas claras desvantagens é o não ser eficiente no cálculo imediato de qualquer termo cuja ordem seja um valor grande ou quando o padrão é apresentado de forma não sucessiva. Além disso, também não promove a capacidade de análise da relação funcional entre cada termo e a sua posição, aspeto chave no pensamento algébrico (KAPUT, 2008; RADFORD, 2008).

A utilização bem sucedida de estratégias de natureza visual implica que as propriedades das figuras sejam identificadas de forma discursiva (DUVAL, 1998). Significa que a disposição espacial dos elementos que compõem a figura é interpretada como uma configuração de objetos que são relacionados através de um invariante, um atributo ou uma propriedade. Nas palavras de Duval (2014), este processo visual é intrinsecamente matemático e envolve discernir e reconhecer unidades figurativas, ou seja, elementos que são matematicamente relevantes numa determinada representação visual. Os alunos que optam por este tipo de abordagem podem fazê-lo de duas formas distintas: identificar conjuntos disjuntos de elementos que são conjugados de modo a obter a figura como um todo, generalização construtiva; detectar a existência de conjuntos sobrepostos, contanto certos elementos mais do que uma vez, que são subsequentemente subtraídos, generalização desconstrutiva (RIVERA; BECKER, 2008). Este último caso envolve um nível de exigência cognitivo superior, aspeto que pode estar o facto de os alunos evidenciarem preferência pela generalização construtiva (BARBOSA; VALE, 2015; RIVERA; BECKER, 2008).

Ao longo do nosso trabalho sobre a descoberta da generalização simbólica nas tarefas de padrões em contextos visuais e concretos, temos percebido que os alunos quando 
discutem uns com os outros recorrem à linguagem verbal mas também aos gestos, na tentativa de explicar o modo como "veem" o padrão, ou seja, quando procuram a estrutura matemática associada à sequência (RADFORD, 2014; WAKEFIELD; CONGDON; NOVAK; GOLDIN-MEADOW; JAMES, 2019). Há pessoas que quando falam, espontaneamente movem as mãos, ou seja, gesticulam, mas estes movimentos não são apenas movimentos das mãos sem significado. A comunicação não-verbal (por exemplo, expressão facial, gestos, movimentos) é reconhecida como uma ferramenta poderosa na aprendizagem, desempenhando um papel único, já que algumas informações não podem ser transmitidas verbalmente, mas sim através por meios não verbais (NEILL, 1991). Os gestos, em particular, servem para expressar ideias e facilitam a aprendizagem em vários domínios (WAKEFIELD et al., 2019), constituindo-se como uma ação que envolve o sistema motor. As descobertas mais recentes da literatura sobre a neurociência na aprendizagem ativa referem que o envolvimento ativo recruta áreas sensoriais e motoras mais intensivamente do que uma aprendizagem mais passiva por parte das crianças (RADFORD, 2014). Muitos conceitos matemáticos são mais bem compreendidos se os alunos tiverem acesso a algum tipo de apoio visual. Assim, os gestos são um excelente meio para prover imagens visuais, sendo reconhecidos como um tipo de comunicação não verbal que complementa os diálogos entre professores e alunos, ajudando o aluno a reter mais informações em relação a uma situação na qual nenhum gesto é realizado (GOLDINMEADOW; KIM; SINGER, 1999). Veja-se um exemplo no contexto da álgebra com um padrão de crescimento. Considere-se a tarefa em que se pede o número de palitos necessários à construção da figura seguinte, mantendo-se a mesma regra, da sequência observada na figura 1 .

Figura 1 - Sequência de quadrados com palitos

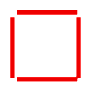

Fig.1

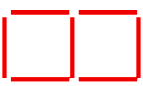

Fig. 2

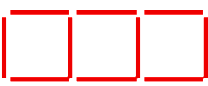

Fig.3

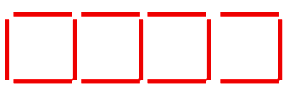

Fig.4

Fonte: Autores

Os alunos podem dar resposta à questão explicando como viram recorrendo a gestos, ou então a representações pictóricas que ajudam a traduzir o que observaram no próprio desenho, como mostra a figura 2. Esta ilustra uma das hipóteses de descobrir a estrutura 
do padrão, na qual os alunos deduzem a fórmula envolvida através de uma relação funcional emergente do modo como viram. A variável (o número do termo) pode surgir na forma algébrica $2 n+(n+1)$ que se transforma em $3 n+1$, ou na forma numérica, em que os números usados são representantes da variável (e.g. o termo de ordem 1000 será 2×1000+1001 palitos), ou, em alternativa, usam a descrição verbal, de acordo com o nível.

Figura 2 - Um modo de ver o padrão

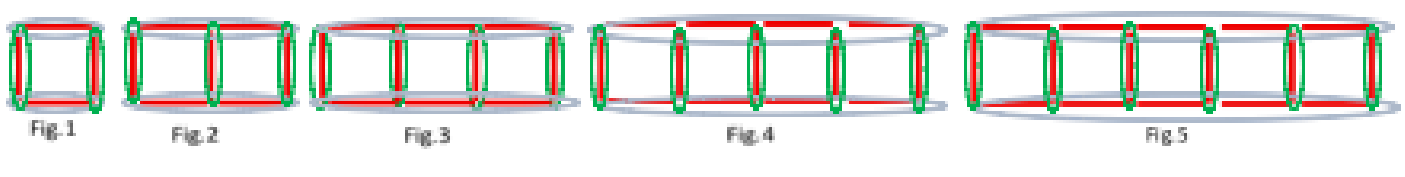

Fonte: Autores

Contudo, há outro aspeto que temos vindo a estudar e que se prende com as múltiplas abordagens que podem emergir da análise de um padrão figurativo. Numa primeira instância, os alunos começam por reproduzir uma sequência (recorrendo a material manipulável ou a um desenho), de modo a que nesse processo de reprodução tenham que recorrer a uma repetição do modo de construção, só depois se incentiva a análise desse processo, para mais facilmente deduzirem a regra usada na construção da sequência.

No exemplo apresentado, pediu-se aos alunos que reproduzissem e continuassem a sequência até ao $5 .^{\circ}$ termo. Apareceram três modos diferentes de desenhar todos os termos. A figura 3 identifica um desses modos, na qual só destacamos a construção, por desenho, do $5 .^{\circ}$ termo da sequência. Um aluno começou por desenhar duas filas paralelas com 5 palitos cada e depois desenhou 6 palitos na vertical, fechando os 5 quadrados do $5 .^{\circ}$ termo da sequência. $\mathrm{Na}$ construção do $6 .^{\circ}$ termo recorreu ao mesmo processo. A partir desta construção descobriu, a partir de um raciocínio funcional, a expressão geral que relaciona o número de palitos com o número da figura e com o modo que utilizou na construção, obtendo a expressão $2 \times 5+6$. A partir daqui conjeturou de imediato a expressão geral $2 x n+(n+1)$. 
Figura 3 - Um modo de desenhar o padrão
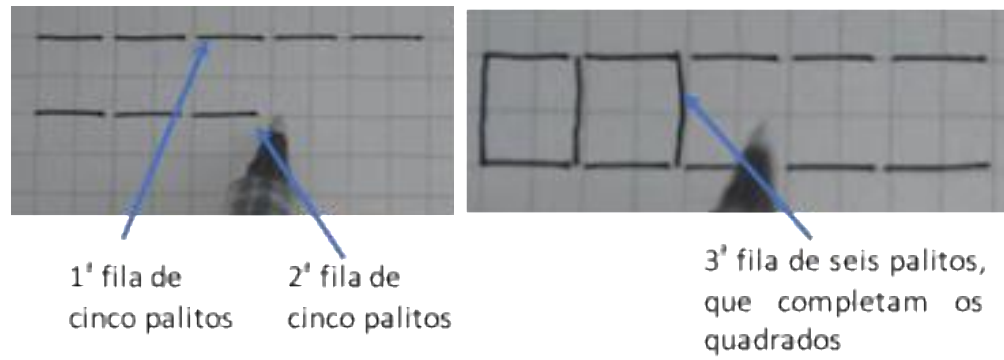

Fonte: Autores

A chamada de atenção para a relação encontrada, levou a que este aluno comentasse: "nunca tinha pensado nisto. Faço assim porque mecanizo e é mais rápido fazer os quadrados, só depois é que olho para o desenho". Ou seja, muitas vezes acontece desenharem de um modo, que não "aproveitam" para gerar a expressão algébrica, voltando a olhar para o desenho de outro modo, ou seja que se traduza numa forma de "mecanizar" diferente. Este é um aspecto importante a explorar sobretudo com os alunos menos visuais, e que está ainda em fase de estudo.

Pode dizer-se que os padrões em contextos figurativos têm um contributo importante na chegada à generalização, permitindo inclusive gerar regras diferentes, ajudando a: reforçar as conexões entre relações aritméticas e espaciais; atribuir significado às regras formuladas; perceber necessidade de formular e validar conjeturas. Geralmente, existem diferentes formas de expressar a relação entre duas variáveis em tarefas desta natureza, tornando-as contextos privilegiados para discutir a aplicação de múltiplas estratégias (múltiplas forma de ver), bem como para explorar a formulação de expressões equivalentes (BARBOSA; VALE, 2015; VALE, 2009).

\section{Metodologia}

Neste estudo procura-se caracterizar o pensamento algébrico de futuros professores do ensino básico (3-12 anos) na resolução de tarefas envolvendo padrões figurativos. Em particular, temos como finalidades identificar as estratégias utilizadas no processo de generalização, categorizando-as em visuais e analíticas, e as dificuldades sentidas.

Tendo por base os objetivos delineados, optou-se por uma metodologia de natureza qualitativa. Participaram neste estudo 43 estudantes do $3 .^{\circ}$ ano de um curso de Licenciatura em Educação Básica (futuros professores de alunos da faixa etária dos 3 aos 12 anos). Os dados foram recolhidos no contexto de uma unidade curricular de 
Didática da Matemática que, entre outros temas, contemplava nos conteúdos programáticos um capítulo dedicado à Álgebra. Neste capítulo foi seguida uma proposta didática (VALE; BARBOSA; BORRALHO; BARBOSA; CABRITA; FONSECA; PIMENTEL, 2011) que se apresenta na figura 4. Os autores definem uma trajetória de aprendizagem, constituída por três fases fundamentais, para o desenvolvimento do pensamento algébrico: Contagens; Sequências; e Problemas. Esta proposta didática atribui um papel fundamental à visualização, privilegiando os contextos figurativos.

Figura 4 - Esquema da proposta didática

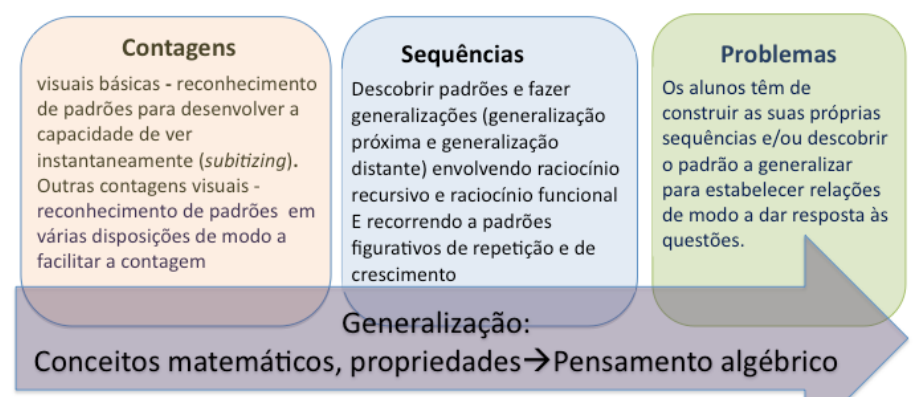

Fonte: Vale et al., 2011

Nesta unidade curricular, começou-se por explorar tarefas de contagens visuais básicas de modo a desenvolver a capacidade de subitizing (ver instantaneamente), evoluindo para contagens visuais em contextos diversificados, para promover o reconhecimento de padrões em várias disposições. Como era esperado, no percurso académico da maioria destes estudantes a visualização, fora do contexto da geometria, não foi muito estimulada, sendo assim esta fase essencial para despertar um outro olhar sobre conceitos já aprendidos. $\mathrm{Na}$ fase seguinte foram exploradas tarefas que envolviam a descoberta de padrões e a construção de generalizações, com padrões de repetição e de crescimento. No entanto, optou-se pelo trabalho mais centrado nos padrões de crescimento pois, apesar de todos os tipos serem necessários ao desenvolvimento do pensamento matemático, os padrões de crescimento conduzem mais naturalmente à descoberta de relações entre duas quantidades variáveis, facilitando o raciocínio visual (RIVERA; BECKER, 2008). Acrescenta-se ainda que foi dada relevância à utilização de tarefas centradas na exploração de padrões figurativos, sendo analisadas diferentes abordagens e representações, de natureza analítica e visual, de modo a promover o pensamento algébrico e a compreensão da estrutura subjacente. $\mathrm{Na}$ última fase foram propostos problemas que implicavam por parte dos estudantes a construção das suas próprias sequências e o estabelecimento de generalizações para chegar à solução. 
Os dados foram recolhidos de forma holística, descritiva e interpretativa e incluíram observações em sala de aula e produções escritas das tarefas propostas. Foram analisados todos os registos escritos produzidos pelos participantes e triangulados com as notas de campo referentes às aulas observadas. A análise destes dados foi orientada por duas grandes categorias: tipo de estratégias (visuais/analíticas); dificuldades sentidas.

\section{Resultados}

Neste artigo optou-se por apresentar os resultados da implementação de uma das tarefas propostas ao longo do estudo (Figura 5). Esta tarefa foi selecionada por permitir ilustrar os resultados mais representativos, referentes às estratégias usadas e às dificuldades sentidas pelos participantes em situações de generalização, próxima (para determinar o termo da sequência pedido é possível utilizar um desenho ou um método recursivo) e distante (os métodos descritos anteriormente não se adequam à resolução deste tipo de questões sendo necessário descobrir uma expressão geral).

Começa-se por apresentar a tarefa:

Figura 5 - Enunciado da tarefa

Observe as três figuras que se apresentam, em que cada figura
tem mais quadrados que a anterior:
1.Sendo estas figuras os três primeiros termos de uma
sequência, descreva a figura seguinte e determine o número
de quadrados que a constituem.
2.Mantendo-se o padrão, determine o número de quadrados
da 10. ${ }^{\mathrm{a}}$ figura. Explique como pensou.
3.Quantos quadrados terá a 100. ${ }^{\mathrm{a}}$ figura? Explique como
pensou.
4. Quantos quadrados terá a figura de ordem n? Explique
como pensou.

Fonte: Autores

Trata-se de um padrão de crescimento linear apresentado num contexto figurativo, no entanto os futuros professores podiam recorrer livremente a estratégias de natureza visual ou analítica na tentativa de generalizar. Como era esperado, surgiram múltiplas abordagens na resolução desta tarefa, por um lado porque o contexto o permitia (usar as figuras ou transformá-las em números), mas também porque alguns participantes poderiam ter mais facilidade em reconhecer as regularidades espacialmente enquanto outros poderiam mostrar preferência pelo reconhecimento analítico (BORROMEOFERRI，2015; GARDNER，1993; KRUTETSKII，1976; PRESMEG，2014). As 
estratégias usadas variaram entre visuais e analíticas, tendo prevalecido as visuais, possivelmente pela influência da proposta didática implementada nas aulas da unidade curricular.

De entre as estratégias analíticas destacaram-se o raciocínio recursivo, na generalização próxima, e a tentativa e erro e o raciocínio funcional, na generalização distante. Nestes casos, os participantes converteram as figuras em números, por norma organizados numa tabela com duas colunas, e identificaram a variação de um termo para o seguinte (+3) até chegarem à $10 .^{a}$ figura. Foram observadas situações de transição para a generalização distante com recurso à tentativa e erro, em que procuraram testar expressões numéricas para os primeiros termos que pudessem evidenciar alguma estrutura: "Para descobrir o número de quadrados da figura 100 usei tentativa e erro até encontrar uma fórmula. Esta sequência varia de 3 em 3 então multipliquei o 3 pelo número da figura adicionando 1, logo tenho 3x100+1, 301 quadrados”. Em alternativa, alguns destes futuros professores reconheceram relações numéricas nos valores plasmados na tabela que deram lugar a uma expressão geral, conseguindo, deste modo, estabelecer uma relação de tipo funcional. Na figura 6 apresenta-se um exemplo de um destes casos, visível na última coluna da tabela.

Figura 6 - Exemplo de raciocínio funcional em contexto analítico

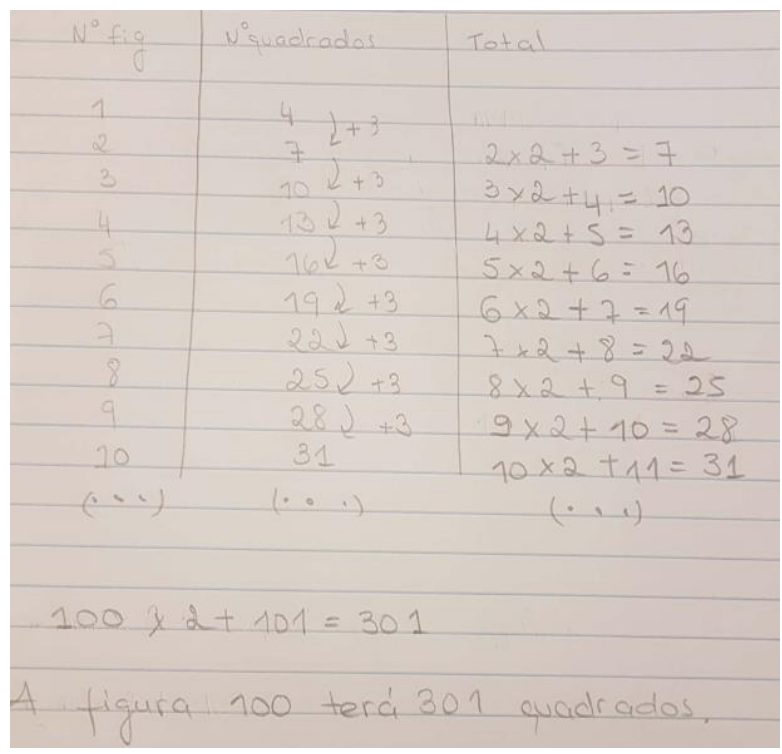

Fonte: Autores

Como se pode observar, neste caso começou por utilizar um raciocínio de tipo recursivo, no entanto, perante uma situação de generalização distante sentiu necessidade 
de mudar de estratégia, uma vez que a relação recursiva não seria útil para termos de maior grandeza. Tendo observado os números da segunda coluna da tabela, conseguiu encontrar relações numéricas, descritas na terceira coluna, que indiciaram a descoberta de uma regra.

Foram identificados seis casos diferentes de estratégias de natureza visual, usados por vários participantes, e que se apresentam na figura 7, agrupados em três situações.

Figura 7 - Diferentes estratégias visuais

(

Fonte: Autores

As estratégias apresentadas em A ilustram a observação dos elementos das figuras por linhas e por colunas, talvez um dos modos mais naturais de percepção visual de uma disposição envolvendo quadrados. Em B são apresentadas as estratégias que surgiram com maior frequência, emergentes da observação de grupos de três elementos em cada uma das figuras. A distinção entre as duas reside na consideração da 1 . $^{\text {a figura como }}$ parte integrante das seguintes ou na identificação de subconjuntos logo a partir da 1. ${ }^{\text {a }}$ figura. Os casos ilustrados em $\mathrm{C}$ foram usados uma única vez, sendo por isso os mais originais. Na primeira situação as figuras foram decompostas em subconjuntos observados na diagonal, dando lugar à formação de grupos de 2 e 3 elementos. Na última situação foram observados grupos de 4 com elementos sobrepostos que 
implicaram um ajuste posterior através da subtração dos quadrados repetidos na contagem. Após a análise das situações descritas, salienta-se que, a maioria dos participantes que recorreu a uma estratégia visual foram capazes de a adaptar às questões de generalização próxima e distante. Estas estratégias permitiram não só chegar à expressão da generalidade em termos simbólicos, através de uma abordagem com significado, estabelecendo um paralelismo entre a regra e o modo de ver a figura, mas também gerar expressões equivalentes. Verificou-se ainda que os futuros professores que optaram por uma resolução desta natureza privilegiaram generalizações construtivas, tendo aparecido apenas um caso $\left(2 .^{a}\right.$ situação em $\left.\mathrm{C}\right)$ representativo de uma generalização desconstrutiva.

Contrariamente ao que sucedeu na generalização próxima, nem todos foram bem sucedidos na generalização distante. Foram observadas dificuldades traduzidas na aplicação de estratégias erradas ou na incapacidade de apresentar uma resposta. Salienta-se que estas situações emergiram apenas na utilização de abordagens de natureza analítica. Para além do recurso sem sucesso à tentativa e erro para a formulação da expressão algébrica, um dos erros que consideramos pertinente destacar, apesar de ter surgido em apenas duas resoluções prende-se com a utilização da proporcionalidade direta quando o padrão não evidencia essa relação (Figura 8). Tratase de uma ferramenta matemática poderosa cuja aplicação pode ser influenciada pela utilização de múltiplos, como acontece na transição do $10 .^{\circ}$ para o $100 .^{\circ}$ termo da sequência, ou pelo trabalho em contextos estritamente numéricos e sem significado (BARBOSA, 2013; LANNIN et al., 2006).

Figura 8 - Exemplo da utilização da proporcionalidade direta

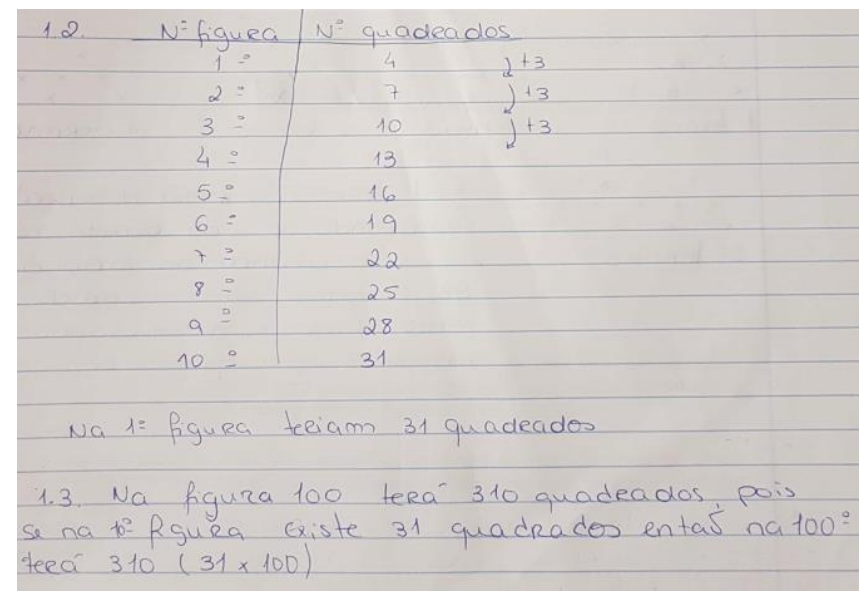

Fonte: Autores 
A transição da generalização próxima para a generalização distante provocou um conflito no raciocínio de alguns destes futuros professores, sendo perceptível uma mudança na escolha da estratégia a usar. Nestas situações começaram sempre por utilizar o raciocínio recursivo para determinar o número de quadrados da 10. a figura e, na generalização distante, optaram por recorrer à tentativa e erro ou à identificação de uma relação funcional através do reconhecimento de relações numéricas generalizáveis. Da análise do trabalho dos participantes foi ainda notória a falta de fluência representacional. Não tendo conseguido chegar à solução a partir de uma estratégia de natureza analítica mostraram-se incapazes de usar as representações visuais na construção da generalização.

Não era objeto de estudo perceber influência dos gestos na procura da estrutura matemática subjacente à sequência, no entanto consideramos que os desenhos realizados pelos participantes, principalmente na resposta à primeira questão, poderão ter tido algum impacto no seu raciocínio. O modo como representaram a $4 .^{\mathrm{a}}$ figura sob a forma de desenho poderá ter dado pistas para a dedução da expressão geral ou pelo menos reforçado a conjetura resultante da observação das primeiras três figuras.

\section{Algumas conclusões}

Quando os alunos dos anos iniciais entram na escola evidenciam um potencial enorme ao nível das capacidades de visualização, de imaginação, de formulação de conjecturas e generalização. Este potencial deve ser explorado pelo professor se pretendemos que estes alunos venham apreciar e a fazer matemática, e não se limitem apenas a treinar um conjunto de técnicas. Os (futuros) professores devem estar atentos ao modo de pensamento dos seus alunos e recorrer a diferentes caminhos e abordagens que permitam explorar as capacidades de cada um, seguindo este procedimento com cada uma das tarefas que utiliza. Acreditamos que os contextos visuais constituem uma mais valia na compreensão da matemática em termos globais e, em particular, no desenvolvimento do pensamento algébrico. Neste artigo são evidenciadas algumas destas potencialidades. Este tipo de tarefas facilita a utilização de múltiplas estratégias de generalização, quer de natureza visual quer de natureza analítica (RADFORD, 2014; RIVERA, 2011; VALE; PIMENTEL; BARBOSA, 2018). A apresentação e o estabelecimento de um paralelismo entre as diferentes abordagens e representações pode ter um contributo importante no aumento do repertório de estratégias do alunos, bem 
como no desenvolvimento da fluência representacional (BIEDA; NATHAN, 2009; BARBOSA; VALE, 2015). A reflexão acerca das estratégias permite ainda perceber que, apesar da multiplicidade de abordagens que podem emergir deste tipo de tarefas, algumas podem apresentar limitações quando se transita da generalização próxima para a generalização distante. É, por isso, fundamental que os alunos conheçam ferramentas que os ajudem a ultrapassar as dificuldades normalmente associadas a esta transição.

Acreditamos que a proposta didática (VALE et al., 2011) utilizada, com o seu forte cunho visual, tem um impacto positivo nos alunos no que refere ao desenvolvimento do pensamento algébrico. A compreensão do significado de expressões numéricas e algébricas, a flexibilidade do pensamento na utilização de diferentes estratégias de generalização, a formulação de expressões equivalentes são alguns exemplos. Através das diferentes fases, a trajetória de aprendizagem delineada na proposta didática facilita a compreensão de conceitos matemáticos poderosos.

\section{Referências}

ARCAVI, A. The Role of Visual Representations in the Learning of Mathematics. Educational Studies in Mathematics, v. 52, p. 215-241, 2003.

BALL, D. L. Prospective elementary and secondary teachers' understanding of division. Journal for Research in Mathematics Education, v. 21, n. 2, p.132-144, 1990.

BARBOSA, A. O contributo da visualização no desenvolvimento do raciocínio funcional. In A. DOMINGOS, I. VALE, M. J. SARAIVA, M. RODRIGUES, M.C. COSTA \& R. FERREIRA, (Eds.), Actas do Encontro de Investigação em Educação Matemática. Penhas da Saúde, Portugal: SPIEM, 2013, p. 51-80.

BARBOSA, A.; VALE, I. Visualization in pattern generalization. Journal of the European Teacher Education Network, v. 10, p. 57-70, 2015.

BIEDA, K. N.; NATHAN, M. J. (2009). Representational disfluency in algebra: Evidence from student gestures and speech. ZDM - The International Journal on Mathematics Education, v. 41, n. 5, p. 637- 650, 2009.

BLANTON, M. Algebra and the elementary classroom: Transforming thinking, Transforming Practice. Portsmouth, NH: Heinemann, 2008.

BLANTON, M.; KAPUT, J. Functional thinking as a route into algebra in the elementary grades. In J. CAI, \& E. KNUTH (Eds.), Early algebraization. A global dialogue from multiple perspectives. Berlin: Springer, 2011, p. 5-23.

BLANTON, M.; KAPUT, J. Characterizing a classroom practice that promotes algebraic reasoning. Journal for Research in Mathematics Education, v.36, n. 5, p. 412-446, 2005 
BORROMEO FERRI, R. Mathematical Thinking styles and their influence on teaching and learning mathematics. Paper presented at the 12th International Congress on Mathematical Education, Seul, Korea. Retrieved in march, 5, 2017 from http://www.icme12.org/upload/submission/1905_F.pdf. 2015.

DUVAL, R. Commentary: Linking epistemology and semio-cognitive modeling in visualization. ZDM - The International Journal on Mathematics Education, v. 46, n. 1, p. 159-170, 2014.

DUVAL, R. Geometry from a cognitive Point of View. In C. MAMMANA \& V. VILLANI (Eds.), Perspectives on the Teaching of Geometry for the 21st Century. Dordrecht, Netherlands: Kluwer Academic Publishers, 1998, p. 37-52.

GARDNER, H. Frames of mind: the theory of multiple intelligences. New York: Basic Books, 1993.

GIANQUITO, M. Visual Thinking in Mathematics. Oxford: Oxford University Press, 2007.

GOLDIN-MEADOW, S.; KIM, S.; SINGER, M. What the teacher's hands tell the student's mind about Math. Journal of Educational Psychology, v. 91, n. 4, p. 720730, 1999.

KAPUT, J. What is algebra? What is algebraic reasoning? In J. KAPUT, D. CARRAHER, \& M. BLANTON (Eds.), Algebra in the Early Grades. New York: Lawrence Erlbaum Associates, 2008, p. 5-17.

KAPUT, J. Teaching and Learning a New Algebra with Understanding. Dartmouth, Massachusetts: National Center, 1998.

KIERAN, C. Overall commentary on early algebraization: Perspectives for research and teaching. In J. CAI \& E. KNUTH (Eds.), Early algebraization. A global dialogue from multiple perspectives. Berlín, Alemania: Springer-Verlag, 2011, p. 557-577.

KRUTETSKII, V. A. The psychology of mathematical abilities in school children. Chicago: University of Chicago Press, 1976.

LANNIN, J., BARKER, D.; TOWNSEND, B. Algebraic generalization strategies: factors influencing student strategy selection. Mathematics Education Research Journal, v. 18, n. 3, p. 3-28, 2006.

MA, L. Knowing and teaching elementary mathematics: teachers' understanding of fundamental mathematics in China and the United States. Hillsdale, NJ: Erlbaum, 1999.

MASON, J. Expressing Generality and Roots of Algebra. In N. BEDNARZ, C. KIERAN \& L. LEE (Eds.), Approaches to Algebra. Dordrecht, The Netherlands: Kluwer Academic Publishers, 1996, p. 65-86.

MOLINA, M. Integración del pensamiento algebraico en la educación básica. un experimento de enseñanza con alumnos de 8-9 años. In M. H. MARTINHO, R. A. T. 
FERREIRA, I. VALE, J. P. PONTE, (Eds), Actas do Encontro de Investigação em Educação Matemática-EIEM. Póvoa de Varzim, Portugal: SPIEM, 2011, p. 27-51.

NEILL, S. Classroom nonverbal communication. London: Routledge, 1991.

NCTM. Principles to actions: ensuring mathematical success for all. Reston: NCTM, 2014.

NCTM. Principles and Standards for School Mathematics. Reston: NCTM, 2000.

PIMENTEL, T. (2011). Um programa de formação contínua e o desenvolvimento do pensamento algébrico de professores do $1 .^{\circ}$ ciclo do ensino básico. In $\mathrm{M}$. $\mathrm{H}$. MARTINHO, R. A. T. FERREIRA, I. VALE, J. P. PONTE, (Eds), Actas do Encontro de Investigação em Educação Matemática-EIEM. Póvoa de Varzim, Portugal: SPIEM, 2011, p. 3-26.

PRESMEG, N. Creative advantages of visual solutions to some non-routine mathematical problems. In S. CARREIRA, N. AMADO, K. JONES \& H. JACINTO, (Eds.), Proceedings of the Problem@Web International Conference: Technology, Creativity and Affect. Faro, Portugal: Universidade do Algarve, 2014, p. 156-167.

RADFORD, L. The progressive development of early embodied algebraic thinking. Mathematics Education Research Journal, v. 26, p. 257-277, 2014.

RADFORD, L. Iconicity and Contraction: A Semiotic Investigation of Forms of Algebraic Generalizations of Patterns in Different Context. ZDM - The International Journal on Mathematics Education, v. 40, n. 1, p. 83-96, 2008.

RIVERA, F. (2011). Toward a Visually-Oriented School Mathematics Curriculum: Research, Theory, Practice, and Issues. Dordrecht, Netherlands: Springer.

RIVERA, F.; BECKER, J. Middle school children's cognitive perceptions of constructive and deconstructive generalizations involving linear figural patterns. ZDM The International Journal on Mathematics Education, v. 40, p. 65-82, 2008.

SWAFFORD, J.; LANGRALL, C. Grade 6 students' pre-instructional use of equations to describe and represent problem situations. Journal for Research in Mathematics Education, v. 31, n. 1, p. 89-112, 2000.

VALE, I. Das tarefas com padrões visuais à generalização. In J. FERNANDES, H. MARTINHO \& F. VISEU (Eds.). Actas XX SIEM - Seminário de Investigação em Educação Matemática. VC: APM, 2009, p. 35-63.

VALE, I.; PIMENTEL, T.; BARBOSA, A. The power of seeing in problem solving and creativity: an issue under discussion. In N. AMADO, S. CARREIRA, \& K. JONES (Eds.), Broadening the scope of research on mathematical problem solving: A focus on technology, creativity and affect. Cham, CH: Springer, 2018, p. 243-272.

VALE, I.; PIMENTEL, T.; BARBOSA, A. Ensinar matemática com resolução de problemas. Quadrante, v. 24, n. 2, p. 39-60, 2015. 
VALE, I.; PIMENTEL, T.; BARBOSA, A.; BORRALHO, A.; BARBOSA, E.; CABRITA, I.; FONSECA, L. Padrões em Matemática - Uma proposta didática no âmbito do novo programa para o Ensino Básico. Lisboa: Texto Editores, 2011.

WAKEFIELD; CONGDON; NOVAK; GOLDIN-MEADOW; JAMES, K. H. Learning math by hand: The neural effects of gesture-based instruction in 8-year-old children. Attention, Perception, \& Psychophysics, v. 81, n. 7, p. 1-11, 2019.

WARREN, E. Generalising the pattern rule for visual growth patterns: Actions that support 8 year olds' thinking. Educational Studies in Mathematics, v. 67, p. 171$185,2008$.

Texto recebido: $31 / 07 / 2019$

Texto aprovado: 23/11/2019 